\title{
Kinetics of electrooxidation of phenol on polycrystalline platinum
}

\author{
Bogusław Pierożyński", Grażyna Piotrowska, Tomasz Mikołajczyk \\ University of Warmia and Mazury in Olsztyn, Department of Chemistry, Faculty of Environmental Management and \\ Agriculture, Plac Eódzki 4, 10-957 Olsztyn, Poland \\ "Corresponding author: e-mail: boguslaw.pierozynski@uwm.edu.pl
}

\begin{abstract}
This work reports on kinetics of phenol electrooxidation reaction $(\mathrm{PhER})$, examined at polycrystalline $\mathrm{Pt}$ electrode in $0.5 \mathrm{M} \mathrm{H}_{2} \mathrm{SO}_{4}$ and $0.1 \mathrm{M} \mathrm{NaOH}$ supporting solutions. Important aspects of PhER kinetics were analysed based on potential-dependent, a.c. impedance-derived values of charge-transfer resistance and capacitance parameters. Special attention was also given to the influence of supporting electrolyte ions on the process of phenol oxidation ( $\mathrm{pH}$ dependence of the PhER), in relation to an important role of anion adsorption on the Pt catalyst surface.
\end{abstract}

Keywords: Phenol electrooxidation; PhER; polycrystalline Pt; Electrochemical impedance spectroscopy.

\section{INTRODUCTION}

Phenolic compounds constitute some of the most important chemical pollutants present in industrial wastewaters. The above concerns numerous important industry sectors, including oil refineries, petrochemical and medicinal drug manufacturing plants. As most phenolic substances are severely toxic and non-biodegradable, they usually cannot be treated with conventional biological purification methods, which are typically employed to treat municipal wastewaters ${ }^{1-5}$.

However, a number of chemical and electrochemical oxidation methods were found to be highly efficient in phenol degradation processes. Electrochemical systems are especially attractive as they usually offer simple apparatus in addition to environmentally-friendly, relatively non-complicated operation and control ${ }^{1,4,6-9}$. Electrochemical oxidation of phenolic compounds has widely been investigated in literature for a number of catalyst anodes, including: noble/semi-noble (e.g. Pt, Ir, $\mathrm{Ru}$ ) and transitional metals ( $\mathrm{Ti}, \mathrm{Sb}, \mathrm{Sn}, \mathrm{Pb}$, etc.), their oxides and mutual compositions for variable $\mathrm{pH}$ and temperature conditions (see for example Refs. 1, 6, 8 and 9 for details).
It is generally accepted ${ }^{1,4,6,9,10}$ that an initial stage of phenol oxidation relates to its conversion to hydroquinone and benzoquinone molecules, which then become further (destructively) oxidized to yield carboxylic acids, and ultimately to form water and carbon dioxide molecules (see the proposed, simplified phenol electrochemical degradation pathway in Fig. 1).

This investigation is concerned with the kinetic aspects of the phenol electrooxidation reaction, where the PhER is studied on a polycrystalline Pt electrode, comparatively under highly acidic (sulphuric acid) and basic (sodium hydroxide) electrolyte conditions.

\section{EXPERIMENTAL}

All solutions were prepared by means of a Direct-Q3 UV ultra-pure water purification system from Millipore (18.2 $\mathrm{M} \Omega \mathrm{cm}$ water resistivity). $0.1 \mathrm{M} \mathrm{NaOH}$ supporting solution was prepared from AESAR, 99.996\% $\mathrm{NaOH}$ pellets, whereas $0.5 \mathrm{M} \mathrm{H}_{2} \mathrm{SO}_{4}$ electrolyte was made up from sulphuric acid of highest purity available (SEASTAR Chemicals). Phenol content (Sigma-Aldrich, >99\%) in both alkaline and acidic supporting solutions was on the order of $1.2 \times 10^{-2} \mathrm{M}$. An electrochemical cell, made of Pyrex glass, was used during the course of this work.

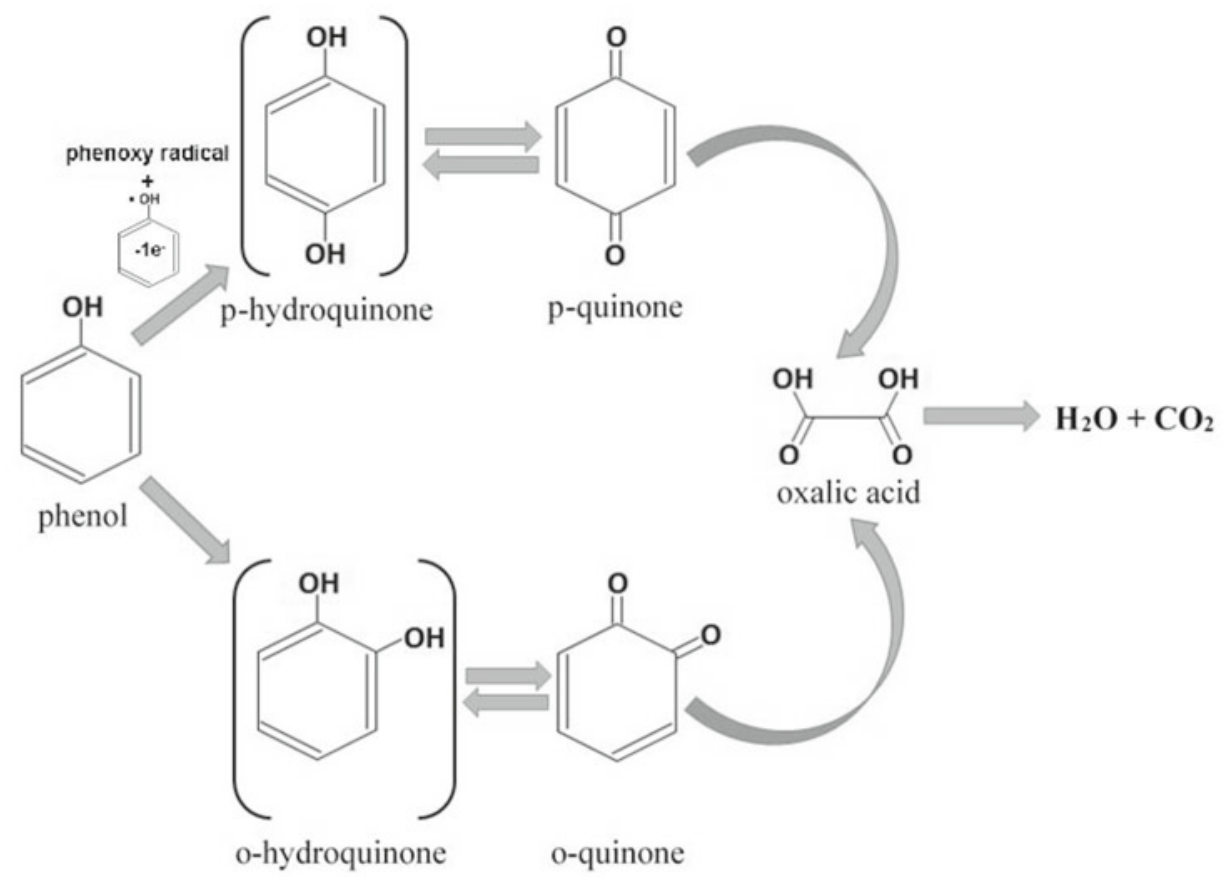

Figure 1. Simplified, schematic representation of electrochemical phenol degradation ${ }^{\mathbf{1}, 3-6,9}$ 
The cell comprised three electrodes: a polycrystalline Pt (1.0 mm diameter wire of $99.9998 \%$ purity, Johnson Matthey, Inc.) working electrode (WE) in a central part, a reversible $\mathrm{Pd}(0.5 \mathrm{~mm}$ diameter wire of $99.9 \%$ purity, Aldrich) hydrogen electrode (RHE) as reference and a Pt $(1.0 \mathrm{~mm}$ diameter wire, $99.9998 \%$ purity, Johnson Matthey, Inc.) counter electrode (CE), both placed in separate compartments. The procedures for cleaning the cell and preparation of the WE/CE/RHE electrodes were as those previously described in other works from this laboratory (e.g. see Ref. 11 for details).

A.c. impedance spectroscopy and cyclic voltammetry techniques were employed in this work. All electrochemical measurements were performed at room temperature by means of the Solartron 12.608 W Full Electrochemical System, consisting of 1260 frequency response analyzer (FRA) and 1287 electrochemical interface (EI). The impedance experiments were carried-out at an a.c. signal of $5 \mathrm{mV}$ and the frequency was swept between $1.0 \times 10^{5}$ and $0.5 \times 10^{-1} \mathrm{~Hz}$, whereas $\mathrm{CV}$ measurements were performed at a sweep-rate of $50 \mathrm{mV} \mathrm{s}{ }^{-1}$. The instruments were controlled by ZPlot 2.9 (Corrware 2.9) software for Windows (Scribner Associates, Inc.), whereas data analysis was performed with ZView 2.9 (Corrview 2.9) software package. The impedance spectra were fitted by means of a complex, non-linear, least-squares immitance fitting program, LEVM 6, written by Macdonald ${ }^{12}$.

\section{RESULTS AND DISCUSSION}

\section{Electrooxidation of phenol in $0.5 \mathrm{M} \mathrm{H}_{2} \mathrm{SO}_{4}$}

The cyclic voltammetric behaviour of phenol (at $1.2 \times 10^{-2} \mathrm{M}$ ) on polycrystalline $\mathrm{Pt}$ electrode in $0.5 \mathrm{M}$ $\mathrm{H}_{2} \mathrm{SO}_{4}$ solution is shown in Figure 2 below. Hence, a major anodic oxidation peak can be observed in the $\mathrm{CV}$ profile over the potential range $1.15-1.35 \mathrm{~V}$ vs. RHE. This peak corresponds to an initial stage of phenol oxidation process (a single-electron charge-transfer step) producing a phenoxy radical cation (Fig. 1), which could further be oxidized to generate p/o-hydroquinone species, finally leading to oxidative destruction of the aromatic ring $1,3,4,6,9,10$. However, some of Pt surface-adsorbed phenoxy radicals could couple to form dimers and further also polymeric compounds, leading to substantial blocking of the Pt surface active sites, thus significantly inhibiting the phenol oxidation process. The above could clearly be observed in the CV profile of Figure 2, where the high potential anodic peak becomes radically suppressed after several electrode cycles carried-out over the potential range $0.0-1.5 \mathrm{~V}$. Furthermore, a broad cathodic reduction feature, observed over the potential range 0.6-0.9 V, could imply partial, but rather very limited reversibility of the PhER. In addition, the low potential voltammetric features (observed at ca. 0.0-0.3 V RHE) are characteristic of significantly inhibited, reversible $\mathrm{H}$ UPD (underpotential deposition) process at $\mathrm{Pt}$, where an onset of cathodic HER (hydrogen evolution reaction) can also be observed (as a sharp spike) just positive to the reversible potential in Figure 2.

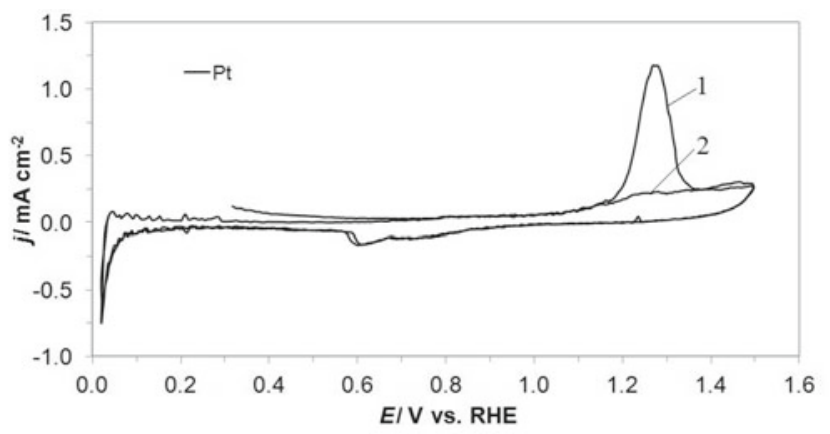

Figure 2. Cyclic voltammogram for electrooxidation of phenol (at $1.2 \times 10^{-2} \mathrm{M}$ ) on poly-crystalline Pt electrode surface, carried-out in $0.5 \mathrm{M} \mathrm{H}_{2} \mathrm{SO}_{4}$ supporting solution at a sweep-rate of $50 \mathrm{mV} \mathrm{s}^{-1}$ (1- and 2- indicate first and second cycles)

The a.c. impedance behaviour of the phenol oxidation process at the polycrystalline Pt electrode, in contact with $0.5 \mathrm{M} \mathrm{H}_{2} \mathrm{SO}_{4}$ solution, is shown in Figure 3 and Table 1. Here, for the examined potential range: $1100-1350$ $\mathrm{mV}$, the impedance spectra exhibit two partial, somewhat depressed semicircles (without a Warburg diffusion element, contrast to the impedance behaviour recorded in Ref. 3, but for lower concentration of phenol, namely $8 \times 10^{-3} \mathrm{M}$ ). The smaller semicircle (see inset to Fig. 3) corresponds to the Faradaic phenol oxidation reaction, whereas the part of a large diameter semicircle, observed throughout the intermediate and low frequencies in Figure 3, is associated with the charge-transfer process accompanying electrosorption of reaction intermediates on the Pt surface (also see Fig. 4a). Thus, the charge-transfer resistance, $R_{\mathrm{F}}$ parameter (see Table 1) corresponds to the oxidation process of phenol on the

Table 1. Resistance and capacitance parameters for the process of phenol electrooxidation (at $1.2 \times 10^{-2} \mathrm{M}$ ) on polycrystalline $\mathrm{Pt}$ electrode surface in contact with $0.5 \mathrm{M} \mathrm{H}_{2} \mathrm{SO}_{4}$ and $0.1 \mathrm{M} \mathrm{NaOH}$ solutions (at room temperature), obtained by fitting the equivalent circuits shown in Figures $4 \mathrm{a}$ and $4 \mathrm{~b}$ to the experimentally-obtained impedance data

\begin{tabular}{|c|c|c|c|c|}
\hline \multicolumn{5}{|c|}{ Electrooxidation of phenol at polycrystalline $\mathrm{Pt}$ in $0.5 \mathrm{M} \mathrm{H}_{2} \mathrm{SO}_{4}$} \\
\hline$E / \mathrm{mV}$ & $R_{\mathrm{F}} / \Omega \mathrm{cm}^{2}$ & $C_{\mathrm{dl}} / \mu \mathrm{F} \mathrm{cm} \mathrm{cm}^{-2} \mathrm{~s}^{\varphi-1}$ & $R_{\text {Ads }} / \Omega \mathrm{cm}^{2}$ & $C_{\text {Ads }} / \mu \mathrm{F} \mathrm{cm}-2 \mathrm{~s}^{\varphi 2-1}$ \\
\hline 1100 & $3.9 \pm 1.1$ & $25.7 \pm 0.7$ & $4.810 \pm 202$ & $84.9 \pm 8.7$ \\
\hline 1150 & $3.9 \pm 1.1$ & $16.2 \pm 0.2$ & $5.883 \pm 158$ & $84.3 \pm 1.2$ \\
\hline 1200 & $4.2 \pm 2.1$ & $20.1 \pm 0.2$ & $4.678 \pm 199$ & $90.4 \pm 2.2$ \\
\hline 1250 & $15.0 \pm 3.0$ & $52.3 \pm 1.4$ & $10.012 \pm 1,146$ & $52.1 \pm 2.1$ \\
\hline 1280 & $19.1 \pm 4.3$ & $52.4 \pm 2.5$ & $7.536 \pm 399$ & $60.1 \pm 2.4$ \\
\hline 1300 & $40.1 \pm 6.5$ & $58.7 \pm 0.1$ & $17.752 \pm 1.775$ & $58.8 \pm 1.2$ \\
\hline 1350 & $40.7 \pm 8.9$ & $73.1 \pm 0.1$ & $11.567 \pm 1.041$ & $39.3 \pm 2.0$ \\
\hline \multicolumn{5}{|c|}{ Electrooxidation of phenol at polycrystalline Pt in $0.1 \mathrm{M} \mathrm{NaOH}$} \\
\hline 1300 & $2.014 \pm 170$ & $73.1 \pm 0.1$ & - & - \\
\hline 1400 & $2.005 \pm 164$ & $45.5 \pm 0.1$ & $4,336 \pm 267$ & $42.1 \pm 1.9$ \\
\hline 1450 & $7.996 \pm 165$ & $54.3 \pm 0.1$ & - & - \\
\hline 1500 & $5.747 \pm 139$ & $57.6 \pm 0.2$ & - & - \\
\hline 1600 & $4.913 \pm 93$ & $52.9 \pm 0.7$ & - & - \\
\hline
\end{tabular}




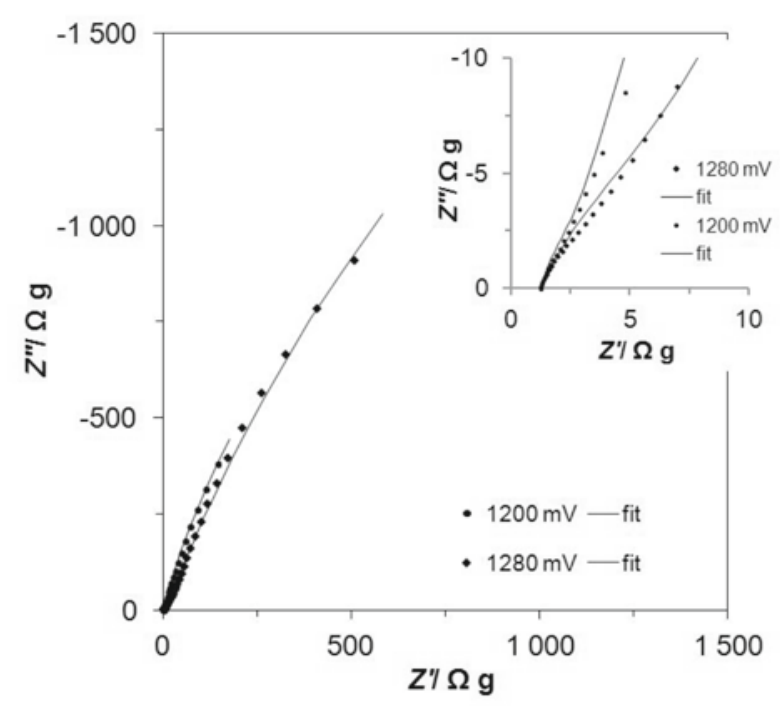

Figure 3. Complex-plane impedance plots for phenol electrooxidation on polycrystalline Pt electrode in contact with $0.5 \mathrm{M} \mathrm{H}_{2} \mathrm{SO}_{4}$ solution, recorded at room temperature for the stated potential values. The solid lines correspond to representation of the data according to the equivalent circuit shown in Figure 4a

Pt electrode surface. It can be seen in Table 1 that the $R_{\mathrm{F}}$ resistance reaches its minimum value of $3.9 \Omega \mathrm{cm}^{2}$ for the potentials of 1100 and $1150 \mathrm{mV}$. Then, for potentials close to that of the voltammetric peak current (ca. $1250 \mathrm{mV}$ ), the $R_{\mathrm{F}}$ parameter significantly increases, reaching finally $40.7 \Omega \mathrm{cm}^{2}$ at $1350 \mathrm{mV}$ (being most likely a result of increasing surface contamination by the reaction by-products, i.e. phenoxy radicals). On the other hand, the adsorption resistance parameter, $R_{\text {Ads }}$ corresponds to the process of electrosorption of the reaction intermediates on the Pt electrode surface. The recorded $R_{\text {Ads }}$ values are dramatically higher than those of the $R_{\mathrm{F}}$ parameter (implying radically slower surface adsorption rates than those of the oxidation process) and fluctuate between $4.678 \Omega \mathrm{cm}^{2}$ (at $1200 \mathrm{mV}$ ) and $17.752 \Omega \mathrm{cm}^{2}$ at $1300 \mathrm{mV}$. Simultaneously, the adsorption capacitance, $C_{\text {Ads }}$ varies between $39.3 \mu \mathrm{F} \mathrm{cm}^{-2} \mathrm{~s}^{\varphi 2-1}$ (at $1350 \mathrm{mV}$ ), and $90.4 \mu \mathrm{F} \mathrm{cm}^{-2} \mathrm{~s}^{\varphi 2-1}$ at $1200 \mathrm{mV}$.

A capacitance dispersion effect (represented by distorted semicircles) can be observed in all Nyquist plots, reported in Figure 3 and later also in Figure 6. Thus, CPE-modified (constant phase element) equivalent circuit models (see Figs. 4a and $4 \mathrm{~b}$ ) were used to represent the electrochemical behaviour of phenol electrooxidation throughout this work. The CPE behaviour is commonly observed in case of inhomogeneous surfaces, displaying surface defects and porosity ${ }^{13-15}$. The double-layer capacitance, $C_{\mathrm{dl}}$ parameter (Table 1) oscillated between $16.2 \mu \mathrm{F}$ $\mathrm{cm}^{-2} \mathrm{~s}^{\varphi 1-1}$ (at $1150 \mathrm{mV}$ ) and $73.1 \mu \mathrm{F} \mathrm{cm}{ }^{-2} \mathrm{~s}^{\varphi 1-1}$, at 1350 $\mathrm{mV}$. It can be seen that for the potentials $1100-1200 \mathrm{mV}$, an average $C_{\mathrm{dl}}$ value comes to $20.7 \mu \mathrm{F} \mathrm{cm}^{-2} \mathrm{~s}^{\varphi 1-1}$, which is very close to $20 \mu \mathrm{F} \mathrm{cm}^{-2}$, a commonly used $C_{\mathrm{dl}}$ value in literature for smooth and homogeneous surfaces ${ }^{16,17}$. On the other hand, significant increase of the $C_{\mathrm{dl}}$ for potentials positive to the voltammetric peak current value implies considerable contribution from the surface electrosorption phenomena. In addition, dimensionless $\varphi_{1}$ and $\varphi_{2}$ parameters ( $\varphi$ determines the constant phase angle in the complex-plane plot, where $0 \leq \varphi \leq 1$ ) of the a)

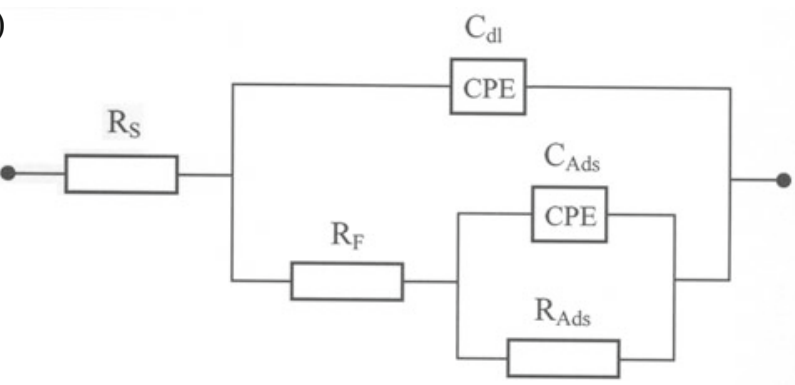

b)

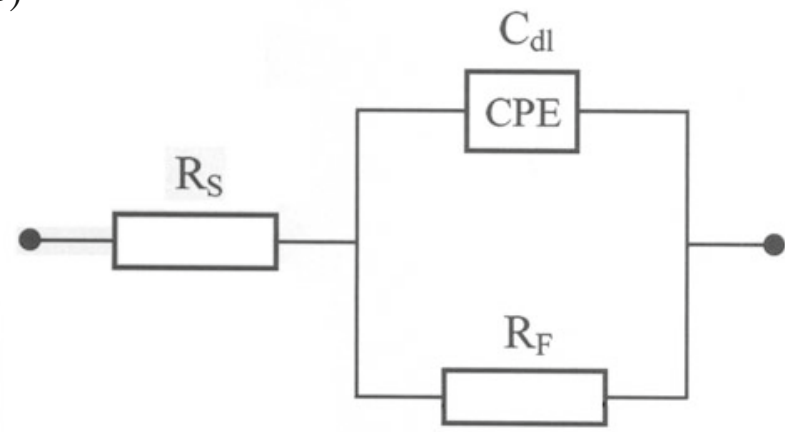

Figure 4. a) Equivalent circuit model for electrooxidation of phenol at polycrystalline Pt electrode, in the presence of adsorbed reaction intermediate. The circuit exhibits a Faradaic charge-transfer resistance, $R_{\mathrm{F}}$, the rate of adsorption (or desorption) of reaction intermediate $\left(R_{\text {Ads }}\right)$ and the component, which contains the contribution of the surface coverage of the adsorbed intermediate, $C_{\text {Ads }}$ (represented here as the constant phase element - CPE). The above components are in a parallel combination with the double-layer capacitance, $C_{\mathrm{dl}}$ (also represented as the CPE), jointly in series with an uncompensated solution resistance, $R_{\mathrm{S}}$; b) As in Figure 4a, but without surface adsorption $\left(R_{\mathrm{Ads}}\right.$ and $\left.C_{\mathrm{Ads}}\right)$ elements

CPE circuits (see again Figs. 4a and 4b) varied between: $0.80-0.95$ and $0.75-0.90$, correspondingly.

\section{Behaviour in $0.1 \mathrm{M} \mathrm{NaOH}$ solution}

Similar cyclic voltammetric behaviour for electrooxidation of phenol (at $1.2 \times 10^{-2} \mathrm{M}$ ) on polycrystalline $\mathrm{Pt}$ electrode was observed in contact with $0.1 \mathrm{M} \mathrm{NaOH}$ supporting solution in Figure 5 below. However, it could be observed in this Figure that compared to the behaviour in acidic solution the phenol oxidation peak in alkaline medium is significantly displaced (by some $170 \mathrm{mV}$ ) towards more positive potentials. In addition, significant increase of anodic current-densities for potentials positive to $c a .1 .8 \mathrm{~V}$ is related to further $\mathrm{Pt}$ surface oxidation processes $(\mathrm{OH} / \mathrm{O}$ species electrosorption phenomena), prior to the Faradaic process of oxygen evolution reaction (OER).

Furthermore, the impedance behaviour of the PhER at the polycrystalline $\mathrm{Pt}$ electrode surface in $0.1 \mathrm{M}$ $\mathrm{NaOH}$ solution, is presented in Figure 6 and Table 1. Here, for the studied potential range: $1300-1600 \mathrm{mV}$ vs. RHE, the impedance spectra exhibit (except for the potential of $1400 \mathrm{mV}$, where electrosorption of reaction intermediates on the Pt surface was clearly identified, see Table 1) a single, large diameter partial semicircle, which corresponds to the Faradaic oxidation reaction (also see Fig. 4b). Hence, the minimum value of the 


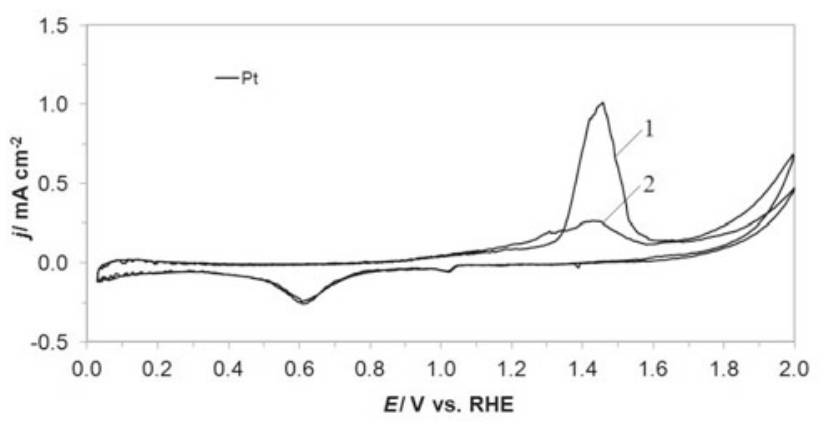

Figure 5. Cyclic voltammogram for electrooxidation of phenol (at $1.2 \times 10^{-2} \mathrm{M}$ ) on poly-crystalline Pt electrode surface, carried-out in $0.1 \mathrm{M} \mathrm{NaOH}$ supporting solution at a sweep-rate of $50 \mathrm{mV} \mathrm{s}^{-1}$ (1- and 2- indicate first and second cycles)

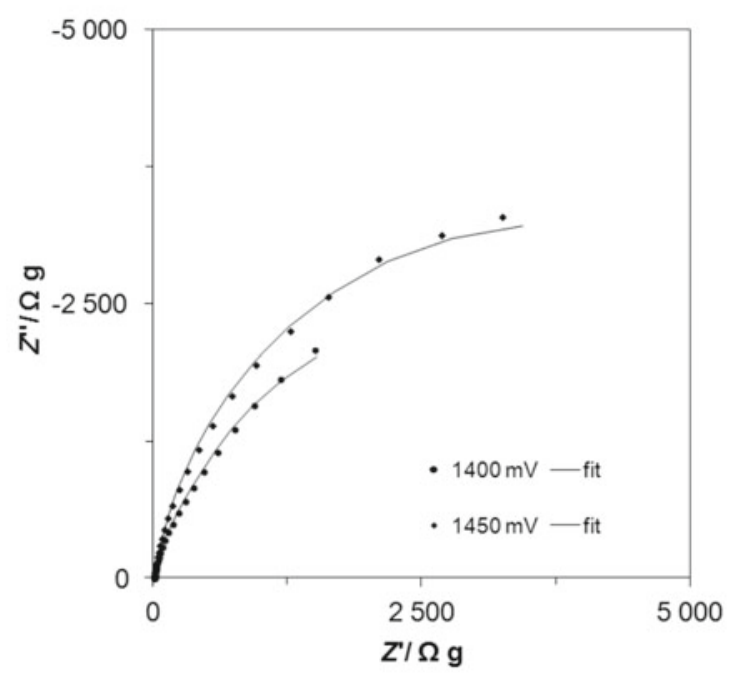

Figure 6. Complex-plane impedance plots for phenol electrooxidation on polycrystalline Pt electrode in contact with $0.1 \mathrm{M} \mathrm{NaOH}$ solution, recorded at room temperature for the stated potential values. The solid lines correspond to representation of the data according to the equivalent circuits shown in Figures $4 \mathrm{a}$ and $4 \mathrm{~b}$ (high frequency features are not shown here)

charge-transfer resistance, $R_{\mathrm{F}}$ parameter (see Table 1 for details) comes to $2.005 \Omega \mathrm{cm}^{2}$ at $1400 \mathrm{mV}$ (close to the peak current-density potential in the $\mathrm{CV}$ profile), which is about 514 times as high as the minimum value of the $R_{\mathrm{F}}$ recorded in acidic medium. These results clearly indicate that the PhER rates are dramatically slower in highly alkaline $\mathrm{NaOH}$ solution than those derived in strongly acidic sulphuric acid medium. The above is most likely the result of restrained formation of the phenoxy radical in addition to the limited extent of phenol adsorption on the Pt surface being in contact with alkaline medium (due to extended $\mathrm{Pt}$ surface coverage by $\mathrm{OH}^{-}$ions, as compared to that of $\mathrm{SO}_{4}{ }^{2-} / \mathrm{HSO}_{4}^{-}$in sulphuric acid at the examined potential range $)^{4,10}$.

In addition, the $C_{\mathrm{dl}}$ parameter exhibited similar behaviour (significant oscillation over the studied potential range) to that observed above in $\mathrm{H}_{2} \mathrm{SO}_{4}$. However, considerably higher values of the double-layer capacitance were recorded in alkaline medium $\left(45.5-73.1 \mu \mathrm{F} \mathrm{cm}{ }^{-2}\right.$ $\mathrm{s}^{\varphi 1-1}$ ), being suggestive of some contribution from the adsorption pseudo-capacitance component. Nevertheless, the effect of increased microscopic roughness of the Pt electrode cannot be completely excluded. Also, dimensionless $\varphi_{1}$ and $\varphi_{2}$ parameters fluctuated between $0.80-0.95$.

\section{CONCLUSIONS}

Electrochemical process of phenol oxidation at polycrystalline Pt surface is strongly $\mathrm{pH}$-dependent. Effective rates of an initial phenol oxidation step are radically faster in strongly acidic solution than those recorded in highly alkaline medium, primarily due to the limited Pt surface sites availability for adsorption of phenol molecules in basic solution (i.e. extended Pt surface coverage by hydroxyl anions). Phenol electrooxidation reaction at the Pt surface proceeds in the presence of adsorbed reaction intermediates (eventually, non-soluble polymeric type compounds), which tend to gradually block the catalyst surface and inhibit the phenol oxidation process. Further kinetic examinations on this topic will be carried-out in order to optimize experimental conditions (reaction $\mathrm{pH}$, temperature, phenol concentration) and the catalyst composition in order to meet the requirements for phenol degradation of industrial wastewaters.

\section{LITERATURE CITED}

1. Torres, R.A., Torres, W., Peringer, P. \& Pulgarin, C. (2003). Electrochemical degradation of $p$-substituted phenols of industrial interest on Pt electrodes. Attempt of a structurereactivity relationship assessment. Chemosphere 50, 97-104. DOI: 10.1016/S0045-6535(02)00487-3.

2. Rajkumar, D. \& Palanivelu, K. (2004). Electrochemical treatment of industrial wastewater. J. Hazard. Mater. B113, 123-129. DOI: 10.1016/j.jhazmat.2004.05.039.

3. Pirvu, C., Banu, A., Radovici, O. \& Marcu, M. (2008). Application of electrochemical impedance spectroscopy (EIS) to study of phenolic films. Rev. Roum. Chim. 53(11), 1007-1015.

4. Lv, G., Wu, D. \& Fu, R. (2009). Performance of carbon aerogels particle electrodes for the aqueous phase electro-catalytic oxidation of simulated phenol wastewaters. J. Hazard. Mater. 165, 961-966. DOI: 10.1016/j.jhazmat.2008.10.090.

5. Yang, X., Kirsch, J., Fergus, J. \& Simonian, A. (2013). Modeling analysis of electrode fouling during electrolysis of phenolic compounds. Electrochim. Acta 94, 259-268. DOI: 10.1016/j.electacta.2013.01.019.

6. Li, X., Cui, Y., Feng, Y., Xie, Z. \& Gu, J. (2005). Reaction pathways and mechanisms of the electrochemical degradation of phenol on different electrodes. Water Res. 39, 1972-1981. DOI: 10.1016/j.watres.2005.02.021.

7. Ma, H., Zhang, X., Ma, Q. \& Wang, B. (2009). Electrochemical catalytic treatment of phenol wastewater. J. Hazard. Mater. 165, 475-480. DOI: 10.1016/j.jhazmat.2008.10.012.

8. Li, M., Feng, C., Hu, W., Zhang, Z. \& Sugiura, N. (2009). Electrochemical degradation of phenol using electrodes of Ti/ $\mathrm{RuO}_{2}-\mathrm{Pt}$ and $\mathrm{Ti} / \mathrm{IrO}_{2}-\mathrm{Pt}$. J. Hazard. Mater. 162, 455-462. DOI: 10.1016/j.jhazmat.2008.05.063.

9. Zhang, C., Jiang, Y., Li, Y., Hu, Z., Zhou, L. \& Zhou, M. (2013).Three-dimensional electrochemical process for wastewater treatment: A general review. Chem. Eng. J. 228, 455-467. DOI: 10.1016/j.cej.2013.05.033.

10. Enache, T.A. \& Brett, A.M.O. (2011). Phenol and parasubstituted phenols electrochemical oxidation pathways. J. Electroanal. Chem. 655, 9-16. DOI: 10.1016/j.jelechem.2011.02.022.

11. Pierozynski, B. (2012). Kinetic aspects of ethanol electrooxidation on catalytic surfaces of $\mathrm{Pt}$ in $0.5 \mathrm{M} \mathrm{H}_{2} \mathrm{SO}_{4}$. Int. J. Electrochem. Sci. 7, 3327-3338. 
12. Macdonald, J.R. (1987). Impedance spectroscopy, emphasizing solid materials and systems. New York: John Wiley \& Sons.

13. Pajkossy, T. (1994). Impedance of rough capacitive electrodes. J. Electroanal. Chem. 364, 111-125. DOI: 10.1016/00220728(93)02949-I.

14. Conway, B.E. (2005). Impedance Spectroscopy. Theory, Experiment, and Applications, Barsoukov, E. \& Macdonald, J.R. (Eds.), Wiley-Interscience, John Wiley \& Sons, Inc., Hoboken, N.J. 4.5.3.8, 494.

15. Pell, W.G., Zolfaghari, A. \& Conway, B.E. (2002). Capacitance of the double-layer at polycrystalline Pt electrodes bearing a surface-oxide film. J. Electroanal. Chem. 532, 13-23. DOI: 10.1016/S0022-0728(02)00676-9.

16. Lasia, A. \& Rami, A. (1992). Kinetics of hydrogen evolution on Ni-Al alloy electrodes. J. Appl. Electrochem. 22, 376-382. DOI: 10.1007/BF01092692s.

17. Chen, L. \& Lasia, A. (1991). Study of the kinetics of hydrogen evolution reaction on nickel-zinc alloy electrodes. $J$. Electrochem. Soc. 138(11), 3321-3328. DOI: 10.1149/1.2085409. 